Original contribution

\title{
4D Flow MRI hemodynamic benchmarking of surgical bioprosthetic valves
}

\author{
Francesco Sturla $^{\mathrm{a}, *, 1}$, Filippo Piatti ${ }^{\mathrm{a}, 1}$, Michal Jaworek ${ }^{\mathrm{b}}$, Federico Lucherini ${ }^{\mathrm{b}}$, \\ Francesca R. Pluchinotta ${ }^{\mathrm{b}, \mathrm{c}, \mathrm{d}}$, Sergii V. Siryk ${ }^{\mathrm{e}}$, Daniel Giese ${ }^{\mathrm{f}}$, Riccardo Vismara ${ }^{\mathrm{b}}$, Giordano Tasca $^{\mathrm{g}}$, \\ Lorenzo Menicanti $^{\mathrm{h}}$, Alberto Redaelli ${ }^{\mathrm{b}}$, Massimo Lombardi ${ }^{\mathrm{c}}$
}

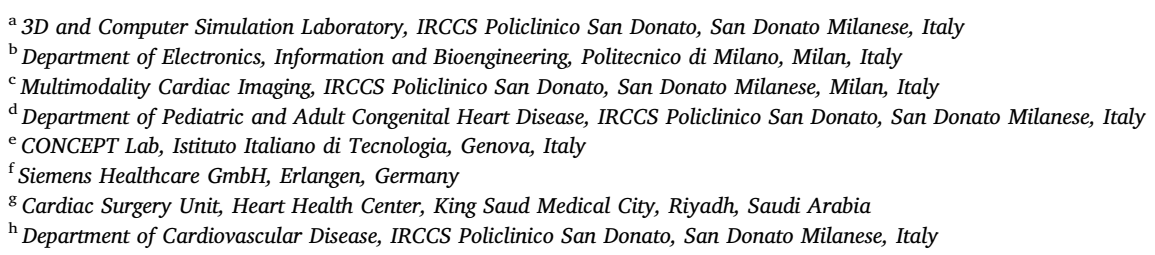

A R T I C L E IN F O

\section{Keywords:}

Bioprosthetic valves

4D Flow MRI

In vitro setting

Hemodynamics

\begin{abstract}
A B S T R A C T
Purpose: We exploited 4-dimensional flow magnetic resonance imaging (4D Flow), combined with a standardized in vitro setting, to establish a comprehensive benchmark for the systematic hemodynamic comparison of surgical aortic bioprosthetic valves (BPVs).

Materials and methods: 4D Flow analysis was performed on two small sizes of three commercialized pericardial BPVs (Trifecta ${ }^{\mathrm{TM}}$ GT, Carpentier-Edwards PERIMOUNT Magna and Crown PRT ${ }^{\circledR}$ ). Each BPV was tested over a clinically pertinent range of continuous flow rates within an in vitro MRI-compatible system, equipped with pressure transducers. In-house 4D Flow post-processing of the post-valvular velocity field included the quantification of BPV effective orifice area (EOA), transvalvular pressure gradients (TPG), kinetic energy and viscous energy dissipation.

Results: The 4D Flow technique effectively captured the 3-dimensional flow pattern of each device. Trifecta exhibited the lowest range of velocity and kinetic energy, maximized EOA $(p<0.0001)$ and minimized TPGs $(p \leq 0.015)$ if compared with Magna and Crown, these reporting minor EOA difference $\mathrm{s}(p \geq 0.042)$ and similar TPGs $(p \geq 0.25)$. 4D Flow TPGs estimations strongly correlated against ground-truth data from pressure transducers; viscous energy dissipation proved to be inversely proportional to the fluid jet penetration.

Conclusion: The proposed 4D Flow analysis pinpointed consistent hemodynamic differences among BPVs, highlighting the not negligible effect of device size on the fluidynamic outcomes. The efficacy of non-invasive 4D Flow MRI protocol could shed light on how standardize the comparison among devices in relation to their actual hemodynamic performances and improve current criteria for their selection.
\end{abstract}

\section{Introduction}

More than 60 million persons are nowadays affected by aortic valve disease [1] with $>250,000$ aortic valves surgically replaced yearly worldwide [2]. Surgical aortic valve replacement, continuously increasing due to progressive population ageing, has revealed a major shift from mechanical valves towards bioprosthetic aortic valves, given the improved hemodynamics of the latter $[1,3]$. Though several aortic bioprosthetic valves (BPV) are currently commercialized to mimic the patient-specific characteristics of the normal native valve, a common challenge is the selection of the optimal valve substitute able to provide the largest effective orifice area (EOA) to blood flow and to minimize the transprosthetic transvalvular pressure gradient (TPG) [4-7]. In particular, proper BPV selection is crucial when implanting stented BPVs in patients with small aortic sizes, frequently revealing high postoperative TPGs and not negligible incidence of patient-prosthesis mismatch (PPM), likely due to a too small EOA in relation to the patient-specific hemodynamic requirements $[3,8,9]$.

Given the lack of uniformity in size labels, commercial valve sizers and suggested sizing strategies $[4,6,10]$, a consistent and

\footnotetext{
* Corresponding author at: 3D and Computer Simulation Laboratory, IRCCS Policlinico San Donato, Via Morandi, 30,20097 San Donato Milanese, Italy.

E-mail address: francesco.sturla@grupposandonato.it (F. Sturla).

${ }^{1}$ The two authors equally contributed to the study.
} 
comprehensive comparison among BPVs is needed to establish reference hemodynamic BPV values of performance under standardized conditions and improve BPV selection criteria. This can be addressed exploiting advanced cardiovascular imaging combined with in vitro testing platforms.

On the one hand, three-dimensional (3D) time-resolved phase contrast magnetic resonance imaging (MRI) with three-directional velocity encoding (4D Flow) can offer a comprehensive insight into blood flow patterns enabling full volumetric coverage of the region of interest, a detailed 3D visualization of the flow pattern and post hoc quantification of several hemodynamic metrics $[11,12]$.

On the other hand, the in vitro setting is the gold-standard approach to consistently investigate the BPVs hemodynamics in a fully controlled environment, through ad hoc system sensorization, under a standardized setup and protocol of measurements, with no influence by confounding in vivo factors $[5,13]$.

Combined with an MRI compatible in vitro set-up, 4D Flow hemodynamic characterization of BPVs has already proved to be feasible on $3 \mathrm{D}$ phantoms $[14,15]$; viability of 4D Flow EOA measurements has already been documented [16] and recent advancements in 4D Flow processing have enabled non-invasive access to relative pressure mapping $[17,18]$. If compared with Doppler echocardiography, which is the method of choice to evaluate BPV function in the clinical setting, 4D Flow has the technical advantage to overcome the bi-dimensional constraint of echo-Doppler imaging and its possible drawbacks due to ultrasound beam misalignment [19].

Hence, within the present study, a dedicated MRI-compatible in vitro system was designed to assess the potential of 4D Flow in providing a consistent benchmark to quantify reference values of hydrodynamic performance in commercialized pericardial aortic BPVs, under a standardized setup and protocol for BPVs hemodynamic comparison. 4D Flow variables were quantified according to state-of-the-art approaches and BPVs compared to detect differences in their fluid-dynamic performance; the reliability of the 4D Flow protocol was finally validated against ground-truth in vitro measurements.

\section{Methods}

\subsection{Tested bioprostheses}

In vitro 4D Flow analysis was accomplished on three commercialized pericardial BPVs: the Trifecta ${ }^{\mathrm{TM}}$ GT (Abbott Laboratories, Irvine, USA), the Carpentier-Edwards PERIMOUNT Magna Ease (Edwards Lifesciences, Irvine, USA), and the Crown PRT ${ }^{\circledast}$ (LivaNova Barnaby, Canada). Specifically, two among the smallest label sizes with comparable stent internal diameter (ID) were selected for each vendor and herein reported as size $A$, ID $=19 \div 20 \mathrm{~mm}$, and size $\mathrm{B}$, ID $=17 \div 18 \mathrm{~mm}$ (Table 1 ). Each BPV was tested over a clinically pertinent flow rate $(\mathrm{Q})$ range of $10 \div 25 \mathrm{l} / \mathrm{min}$, with a $5 \mathrm{l} / \mathrm{min}$ increment.

Table 1

Tested small BPV models and corresponding dimensions.

\begin{tabular}{llllll}
\hline Model & Size & Label size & Stent ID & Stent OD & $\begin{array}{l}\text { Sewing ring } \\
\text { ED }\end{array}$ \\
& & & & & 26 \\
Trifecta $^{\text {TM }}$ GT & A & 21 & 19 & 21 & 24 \\
& B & 19 & 17 & 19 & 24 \\
PERIMOUNT Magna & A & 21 & 20 & 21 & 26 \\
Ease & B & 19 & 18 & 19 & 24 \\
Crown PRT & A & 23 & 19 & 22.7 & 26 \\
& B & 21 & 17.3 & 20.7 & 23 \\
\hline
\end{tabular}

All dimensions are expressed in $\mathrm{mm}$.

$\mathrm{ID}=$ internal diameter; $\mathrm{OD}=$ outer diameter; $\mathrm{ED}=$ external diameter.

\subsection{Experimental set-up}

The dedicated and MR-compatible in vitro test bench (Fig. 1) consisted of a centrifugal pump (BM04APP, Savino-Barbera, Brandizzo, TO, Italy) pumping a blood mimicking fluid (water-glycerol solution, $\mu=3 \mathrm{cP}$ ) towards the inlet port of a paradigmatic 3D-printed aortic root (AR). Each BPV was adequately housed into the AR by compressing axially the suture ring, with AR longitudinal axis parallel to the headfoot direction and avoiding any distortion of the BPV struts; a cylindrical phantom was positioned alongside to increase the signal received by the MR scanner. The entire phantom was placed on the MRI bed and firmly fixed using the MRI thorax coil; additional plastic ties were employed to prevent any motion of the hydraulic connections. A transit-time flow meter (HT110R, Transonic Systems, Ithaca, NY, USA) with a 3/8 flow probe monitored $Q$ downstream of the centrifugal pump. The circuit was then closed into the reservoir, which was connected to the AR outlet port. We assembled the connections between the hydraulic line and the 3D-printed AR employing non-ferromagnetic materials while the pump and the electronic hardware were positioned outside the MR scanner room [20]. Pressures were measured by pressure transducers (140 PC series, Honeywell Inc., Morristown, NJ, USA) at four pre-defined locations along the longitudinal axis of the system (Fig. 1): one (P0) located $25 \mathrm{~mm}$ upstream the aortic annulus and three downstream $(\mathrm{x}=15,40$ and $115 \mathrm{~mm}$ with $\mathrm{x}=0$ referring to AR annulus). The first and the last downstream pressure ports (P1 and P3) were used to calculate TPG $\mathrm{MAX}_{\mathrm{M}}$ and $\mathrm{TPG}_{\text {net }}$ : the former as the pressure drop between $\mathrm{P} 0$ and $\mathrm{P} 1$ and the latter as the pressure drop between P0 and P3. The central pressure port (P2) was available as additional pressure information for comparison with 4D Flow data. The transducers, as other electronic equipment, were placed outside the MRI room and connected with the pressure ports by $1 / 4$ " Tygon tubing. The flow and pressures were acquired concomitantly with 4D Flow data.

\subsection{D Flow analysis}

A prototype 4D Flow sequence (Fig. 2A) was used on a Magnetom Aera $1.5 \mathrm{~T}$ (Siemens Healthcare, Erlangen, Germany) adopting the following MR parameters: field of view up to $270 \times 187 \times 93.6 \mathrm{~mm}$, isotropic $1.3 \times 1.3 \times 1.3 \mathrm{~mm}$ spatial resolution, echo time $2.8 \div 3.7 \mathrm{~ms}$, flip angle $8^{\circ}$ (please refer to Tables S1 and S2 for the specific setting of each tested 4D Flow sequence).

Velocity encoding was adjusted to minimize velocity aliasing based on long-axis MRI scout sequences (Table S3). Specifically, long-axis velocity encoding range $\left(\mathrm{VENC}_{\text {long }}\right.$ ) was set $10 \%$ higher than the expected maximum velocity [11] to minimize velocity aliasing using MRI scout sequences; in the direction perpendicular to the main flow vector, isotropic in-plane VENC $\left(\mathrm{VENC}_{\text {in-plane }}\right)$ was set equal to $1 / 3$ of $\mathrm{VENC}_{\text {long }}$ to facilitate the analysis of flow regions with low velocity around BPV. 4D Flow data were processed through in-house Matlab (The MathWorks Inc., Natick, MA, USA) code [21] and using the open-source ParaView (Kitware Inc., Clifton Park, NY, USA) environment (Fig. 2B). For each flow rate, the 4D Flow volume was averaged voxel-wise over 5 consecutive acquisitions (overall duration of $3 \mathrm{~min}$ ) to improve signal-tonoise ratio.

For each BPV, the post-valvular 3D flow was characterized by means of six velocity isosurfaces (i.e., the 3D region of space characterized by the same velocity magnitude value) extracted from the $65^{\text {th }}, 70^{\text {th }}, 75^{\text {th }}$, $80^{\text {th }}, 85^{\text {th }}$ and $90^{\text {th }}$ percentile of the maximum velocity magnitude $\left(\mathrm{V}_{\mathrm{MAX}}\right)$, whose location within the $90^{\text {th }}$ isosurface identified the BPVspecific vena contracta, i.e., the peak velocity within the 3D velocity field. The jet distance $\left(J_{D}\right)$, a metric of jet penetration within the in vitro system, was computed as the distance between the highest point of each isosurface and the valvular housing.

\subsubsection{EOA calculation}

4D Flow EOA estimation, i.e., the cross-sectional area of the 


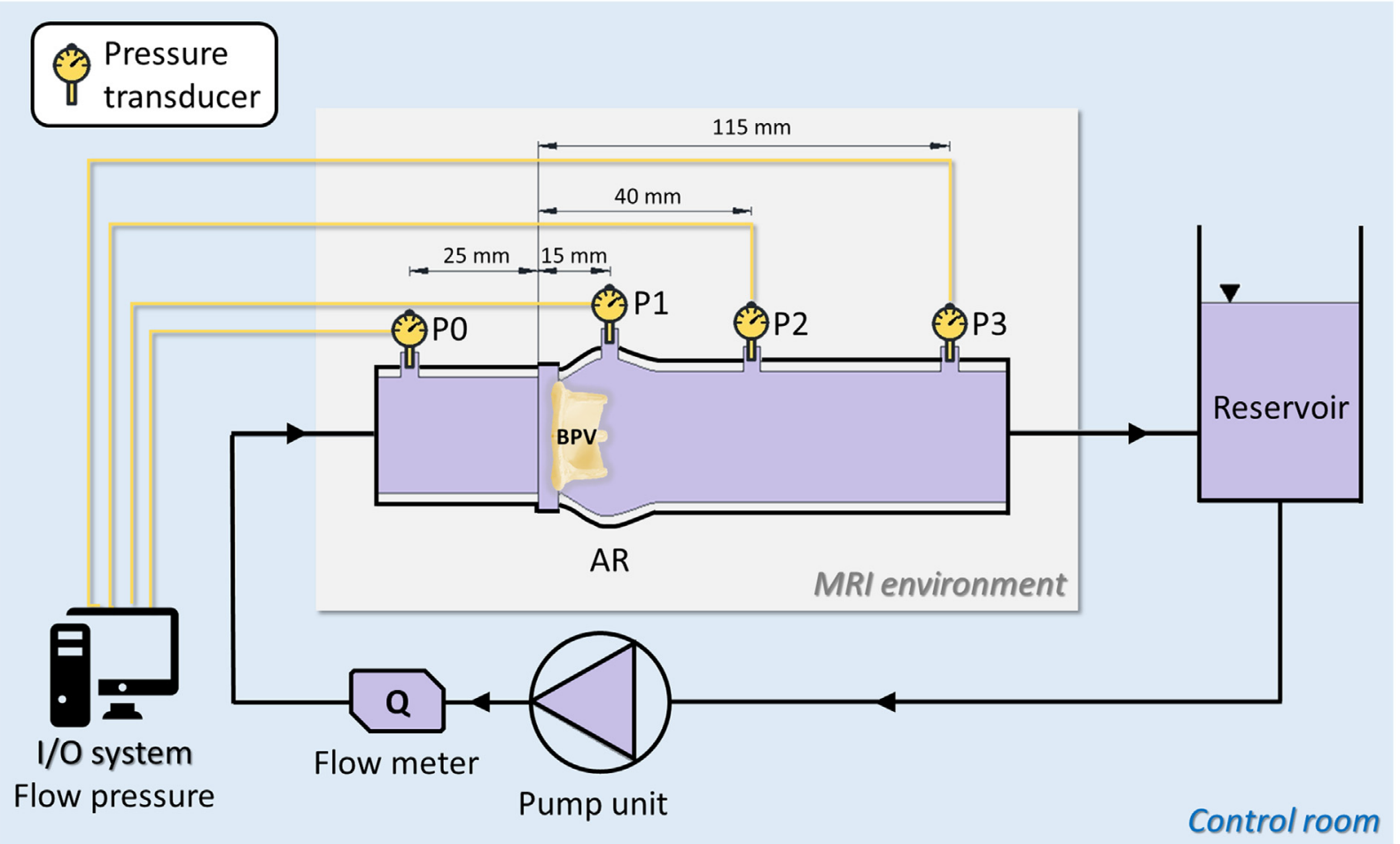

Fig. 1. In vitro experimental set-up.

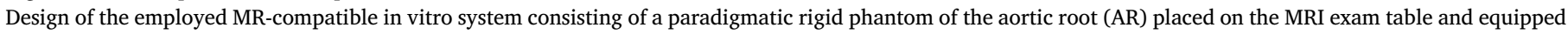

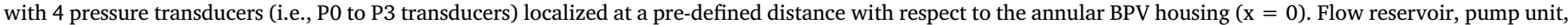

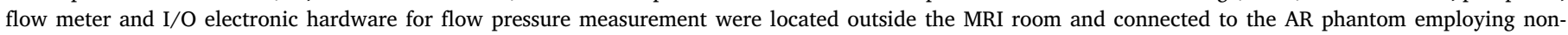
ferromagnetic materials.

compressed bloodstream at the level of vena contracta, was accomplished according to single plane velocity truncation (SPVT) and jet shear layer detection (JSLD) methods [16,22].

The JSLD method is based on the acoustical source term (AST), computed from the velocity field [23,24], and providing an enhanced visualization of flow jet separation from recirculating flow downstream from the aortic valve. The AST scalar field scales the vorticity field by the velocity magnitude as:

$A S T=\nabla \cdot(\omega \wedge v)$

where $\boldsymbol{v}$ is the velocity field, $\boldsymbol{\omega}$ the corresponding vorticity field, $\nabla$ the nabla operator and $\wedge$ the wedge product. The minimum cross-sectional area of the AST field is detected at the peak velocity position downstream of the aortic valve, thus identifying the vena contracta. An automated contour detection algorithm was employed to extract the AST cross-sectional shear layer profile, which internal area corresponds to $\mathrm{EOA}_{\text {JSLD }}$. Specifically, the AST cross-section located at the vena contracta (Fig. 3A) was sampled on a polar coordinate system $(\vartheta, \rho)$ to extract normalized profiles, on which an automated peak detection algorithm was employed to identify the two highest peaks (Fig. 3B), i.e., $\left(\vartheta_{1}, \rho_{1}\right)$ and $\left(\vartheta_{1}+\pi, \rho_{2}\right)$, respectively. Peaks identification was accomplished with a $\vartheta$ sampling rate equal to $5^{\circ}$; $\mathrm{EOA}_{\mathrm{JSLD}}$ was finally defined as the area enclosed by all the detected points.

In the SPVT method, the normalized 3D velocity map is truncated with a threshold of $0.65\left(\lambda_{65}\right)$, which defines the velocity isosurface characterized by the same velocity magnitude corresponding to the $65^{\text {th }}$ percentile of velocity peak $\left(\mathrm{V}_{\mathrm{MAX}}\right)$. At the level of the vena contracta cross-section, pixel inside the $\lambda_{65}$ threshold are counted to estimated $\mathrm{EOA}_{\mathrm{SPVT}}$.

Both $\mathrm{EOA}_{\mathrm{SPVT}}$ and $\mathrm{EOA}_{\mathrm{JSLD}}$ were compared with experimental values $\left(\mathrm{EOA}_{\mathrm{Gorlin}}\right)$ derived from the Gorlin formula, which takes the pressure recovery phenomenon into account according to the corrected Gorlin formula [25]:
$E O A_{\text {Gorlin }}=\frac{Q}{50 \sqrt{T P G_{M A X}}}$

where $Q$ is expressed in $\mathrm{ml} / \mathrm{s}$ and $T P G_{M A X}$ is the maximum transvalvular pressure gradient measured in $\mathrm{mm} \mathrm{Hg}$ by the pressure transducer positioned at port P1 (Fig. 1). BPVs were also compared in terms of the performance index $(\mathrm{Pi})$, defined as the ratio between each EOA and the BPV-specific internal nominal area [13]:

$P i=\frac{E O A}{\pi \frac{I D^{2}}{4}}$

where $I D$ is the internal BPV diameter and the internal nominal area was assumed circular. In all the tested BPVs, Pi was computed according to the different methods of EOA estimation, i.e., $\mathrm{EOA}_{\mathrm{SPVT}}$, $\mathrm{EOA}_{\mathrm{JSLD}}$ and $\mathrm{EOA}_{\mathrm{Gorlin}}$.

\subsubsection{Transvalvular pressure gradients}

The 3D relative pressure field was extracted from the discrete velocity field yielded by the 4D Flow sequence along the longitudinal centerline of the in vitro set-up (i.e., the longitudinal axis of the in vitro phantom) to quantify the maximum transvalvular pressure gradient $\left(T P G_{M A X}\right)$, the net transvalvular pressure gradient $\left(\mathrm{TPG}_{\text {net }}\right)$ and their difference, i.e., pressure recovery. The pressure gradient $\boldsymbol{b}\left(\mathrm{N} \cdot \mathrm{m}^{-3}\right)$ was expressed rearranging the Navier-Stokes equation as $[17,26]$ :

$\boldsymbol{b}=-\rho \frac{\partial \boldsymbol{v}}{\partial t}-\rho(\boldsymbol{v} \cdot \nabla \boldsymbol{v})+\mu \nabla^{2} \mathbf{v}$

where $t$ is the time, $\rho$ the density $\left(1060 \mathrm{~kg} \cdot \mathrm{cm}^{-3}\right)$ and $\mu$ the viscosity $\left(4 \cdot 10^{-3} \mathrm{~N} \cdot \mathrm{s} \cdot \mathrm{m}^{-2}\right)$. First order and second order spatial velocity derivatives were calculated with finite differences, characterized by a variable approximation order depending on the position of the considered point within the domain: e.g., from 1st order for boundary points to 4th order for bulk flow points. Time-dependency was 

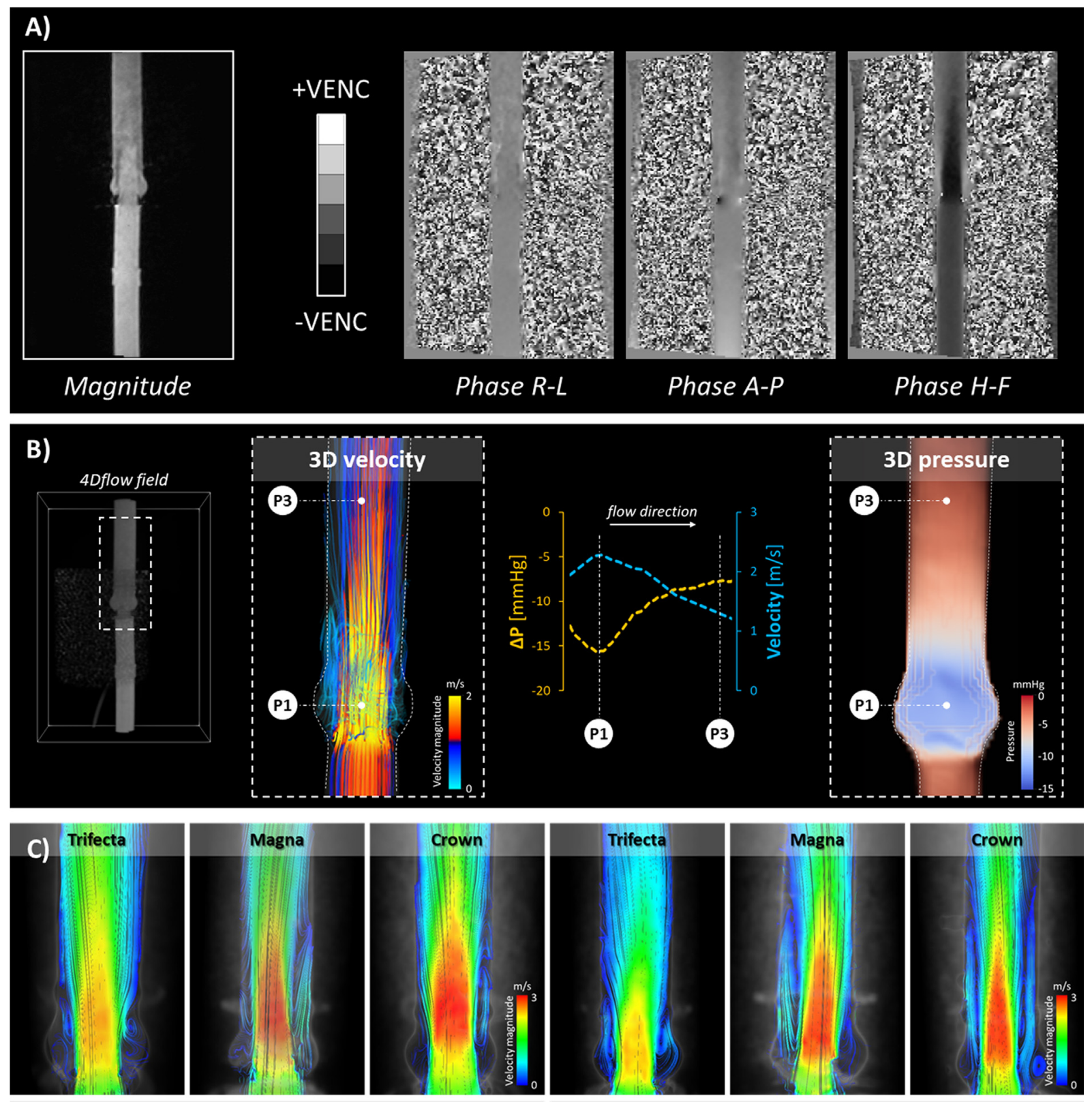

Size A

Size B

Fig. 2. 4D Flow analysis.

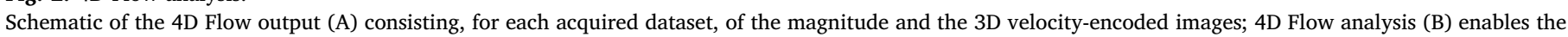

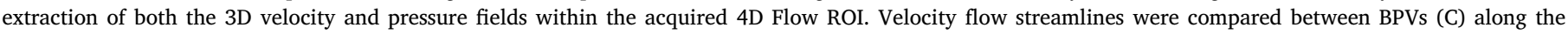
longitudinal axis of the 3D-printed AR model, herein visualized at $\mathrm{Q}=25 \mathrm{l} / \mathrm{min}$.

neglected due to the steady condition of the flow field.

The Pressure Poisson Equation (PPE) was defined from the divergence of Eq. (3) as:

$\nabla^{2} p=\nabla \cdot \boldsymbol{b}$

Relative pressure maps were finally computed by integration of the computed gradients through a multigrid finite element solver [17], already validated against gold-standard estimations provided by computational fluid dynamics models [20,27]. Specifically, the numerical PPE solution resulted in the relative pressure field $p$ and a zero-pressure reference was imposed at the same location of the experimental P0 pressure port (Fig. 1).

Subsequently, the velocity field was sampled along the longitudinal centerline coordinate $(x)$ of the in vitro phantom and the velocity points corresponding to P0 (i.e., $v_{P 0}$ ) and to P1 (i.e., $v_{P 1}$ ) ports (Fig. 1) were used to compute the transvalvular pressure drop $\Delta p_{\text {valve }}$ according to the modified Bernoulli equation:

$\Delta p_{\text {valve }}=4 \cdot\left(v_{P 1}^{2}-v_{P 0}^{2}\right)$

To minimize possible peaks underestimation due to post-valve related artifacts [28], the difference $\Phi_{p}$ between the absolute minimum $\left(p_{\text {min }}\right)$ of the pressure field $p$ and $\Delta p_{\text {valve }}$ was applied as a shift to correct the pressure field, thus obtaining the corrected pressure field $p_{c o r r}(x)$ :

$p_{\text {corr }}(x)=p(x)+\Phi_{p}=p(x)+\left(\left|p_{\min }\right|-\Delta p_{\text {valve }}\right)$

with $\mathrm{TPG}_{\mathrm{MAX}}$ and $\mathrm{TPG}_{\mathrm{net}}$ corresponding to $p_{\text {corr }}(x=P 1)$ and $p_{\text {corr }}(x=P 3)$, respectively. 4D Flow-derived TPG estimations were compared against ground-truth measurements directly taken from the pressure transducers. 


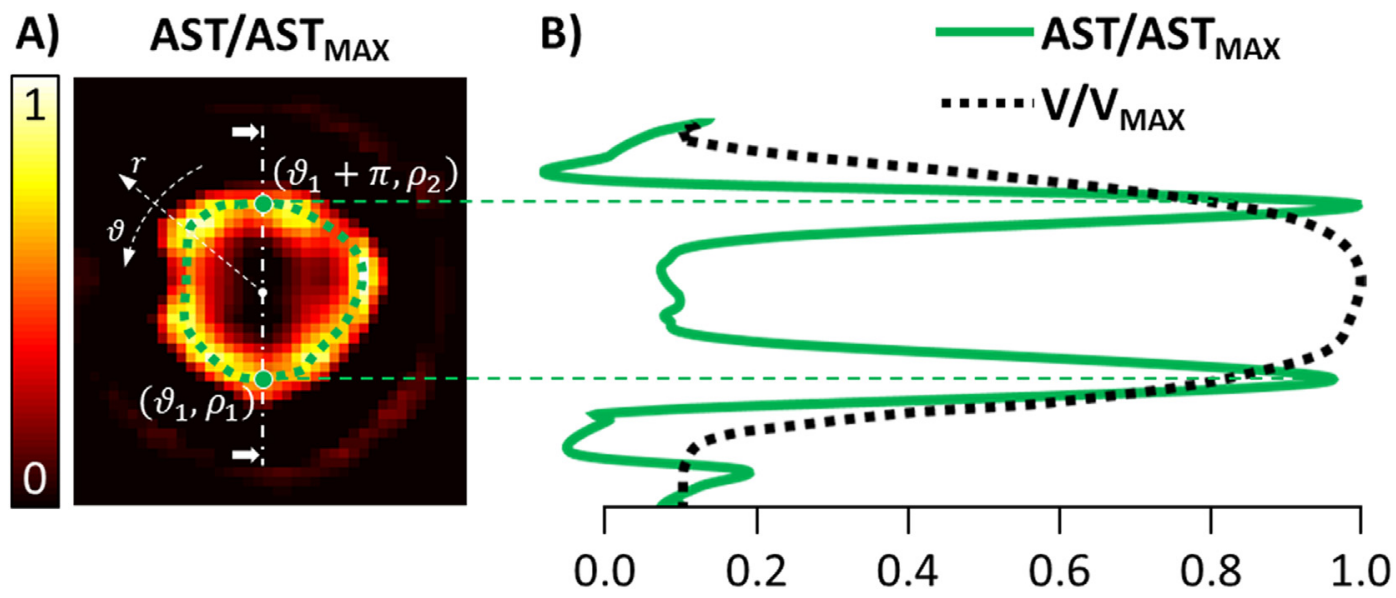

Fig. 3. EOA jet Shear layer detection method.

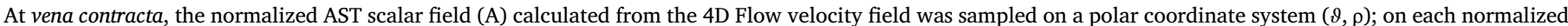

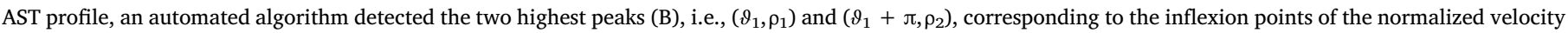

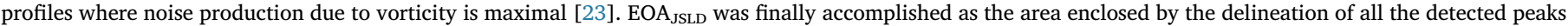
(green dotted line). (For interpretation of the references to color in this figure legend, the reader is referred to the web version of this article.)

\subsubsection{Kinetic energy and viscous energy loss rate}

The post-valvular 4D Flow volume, up to $10 \mathrm{~cm}$ downstream of each BPV along the longitudinal axis of the AR phantom, was considered to quantify the kinetic energy (KE), the energy conversion of static pressure into dynamic pressure to accelerate blood to the vena contracta, for each 4D Flow voxel as [29]

$K E=\sum_{i=1}^{N_{\text {voxels }}} \frac{1}{2} \rho V_{i} v_{i}^{2}$

where $V_{i}$ is the ith voxel volume and $v$ the velocity magnitude.

In addition, the rate of instantaneous viscous dissipation $\left(\dot{E}_{L}\right)$, also referred as viscous energy loss and expressed in power unit [30], was calculated within the post-valvular 4D Flow volume of interest. $\dot{E}_{L}$, which is a 4D Flow-derived hemodynamic metric of irreversible power loss due to viscous effects, was estimated by the product of the dynamic blood viscosity $(\mu)$ and the voxel-wise integral of a viscous dissipation function $\phi_{V}$, as:

$\dot{E}_{L}=\mu \sum_{i=1}^{N_{\text {voxels }}} \phi_{v} V_{i}$

with $N_{\text {voxels }}$ the total number of voxels within the region of interest. The viscous dissipation function $\phi_{v}$ per unit volume [30,31], expressed in $\mathrm{s}^{-}$ ${ }^{2}$, can be obtained reformulating the viscous portion of the incompressible Navier-Stokes energy equation as:

$\phi_{V}=\frac{1}{2} \sum_{i=1}^{3} \sum_{j=1}^{3}\left[\left(\frac{\partial v_{i}}{\partial x_{j}}+\frac{\partial v_{j}}{\partial x_{i}}\right)-\frac{2}{3}(\nabla \cdot v) \delta_{i j}\right]^{2}$

where $i$ and $j$ represent the principal orthogonal velocity directions (i.e., $\mathrm{x}, \mathrm{y}$ and $\mathrm{z}$, respectively), $\delta_{i j}$ is the Kronecker delta $\left(\delta_{i j}=1\right.$ for $\mathrm{i}=\mathrm{j}$ and $\delta_{i j}=0$ for $\mathrm{i} \neq \mathrm{j}$ ) and $\nabla \cdot \boldsymbol{v}$ is the divergence of the velocity field.

\subsubsection{Statistical analysis}

Continuous variables, expressed as mean \pm SD after Shapiro-Wilk normality tests, were compared using two-way analysis of variance considering BPV type and Q as independent factors; the Bonferroni correction was used in post hoc analysis. Correlations were assessed with linear regression; differences in reported EOA and TPG estimations (e.g., between 4Dflow and transducers measurements) were assessed through Bland-Altman plots. Statistical analyses were performed using GraphPad Prism 7 (GraphPad Software Inc., La Jolla, CA, USA); a $p$ value $<0.05$ was considered significant.

\section{Results}

\subsection{BPV differential 3D flow pattern}

4D Flow acquisitions were successfully acquired within the experimental environment for all BPVs and testing conditions (Fig. 2C). Flow rate measurements (Fig. 4A, Table S4), taken from the flow meter on the in vitro system, revealed excellent agreement $\left(r^{2}=0.999\right)$ with the 4D Flow MRI measurements; paired $t$-test comparison reported negligible differences $(p=0.20)$ and percentage relative change ranged between $-3.2 \%$ (Crown size B, $10 \mathrm{l} / \mathrm{min}$ ) and $+2.7 \%$ (Trifecta size A, $20 \mathrm{l} / \mathrm{min}$ ) with respect to the corresponding 4D Flow measurement.

The analysis provided a clear 3D evaluation of the velocity isosurfaces (Fig. 4B), accurate localization of vena contracta and comprehensive evaluation of the velocity field downstream of each BPV (Fig. S1).

For each valve size, $\mathrm{V}_{\mathrm{MAX}}$ linearly increased with $\mathrm{Q}$ and showed a similar trend in Magna and Crown while it was significantly lower $(p<0.0001)$ in Trifecta (Fig. 4C). Mean jet distance proved to be unaffected by $\mathrm{Q}(p>0.8)$; minor differences among sizes A and B were observed, especially at $25 \mathrm{l} / \mathrm{min}$, in Magna and Crown, with the latter showing a higher jet penetration $(57.7 \pm 11.1 \mathrm{~mm}$, size B). By contrast, valve size had a strong impact on the downstream flow of Trifecta, which exhibited the shortest ( $36.6 \pm 10.6 \mathrm{~mm}$, size B) and the longest $(59.7 \pm 16.4 \mathrm{~mm}$, size A) jet distances depending on the valve size (Fig. 4D).

\subsection{Effective orifice area}

The overall EOA shape (Fig. 5A) was almost circular for Trifecta, markedly trilobal for the Magna and triangular for Crown BPV; as a result, Trifecta EOA was maximized $(p<0.0001)$ for both A and B sizes (Fig. 5A). Magna and Crown were comparable for size B (EOA JSLD, $p=0.098$ ) while differences were not negligible (EOA JSLD,$p=0.042$ ) for size A. The two methods of EOA estimation from $4 \mathrm{D}$ Flow reported excellent agreement $\left(\mathrm{r}^{2}=0.96\right.$, Fig. 5B) with almost no bias $(\mathrm{b}=-0.8 \%$ ) and low variation (Bland-Altman limits of agreement between $-8.8 \%$ and $7.2 \%$, Fig. $6 \mathrm{~A}$ ). Both methods exhibited a strong correlation against $\mathrm{EOA}_{\mathrm{Gorlin}}\left(\mathrm{r}^{2}=0.84\right.$ for both $\mathrm{EOA}_{\mathrm{SPVT}}$ and $\left.\mathrm{EOA}_{\mathrm{JSLD}}\right)$ with comparable biases $\left(\mathrm{b}=-2.6 \%\right.$ for $\mathrm{EOA}_{\mathrm{SPVT}}$ and $\mathrm{b}=-3.3 \%$ for $\left.\mathrm{EAO}_{\text {JSLD }}\right)$ and similar limits of agreements $(-15.9 \% \div 10.7 \%$ for $\mathrm{EOA}_{\mathrm{SPVT}}$ and $-17.8 \% \div 11.1 \%$ for $\mathrm{EOA}_{\mathrm{JSLD}}$ ). Trifecta maximized $\mathrm{Pi}$ for each valve size ( $p<0.0001)$, with overall $\mathrm{Pi}$ values ranging 
A)

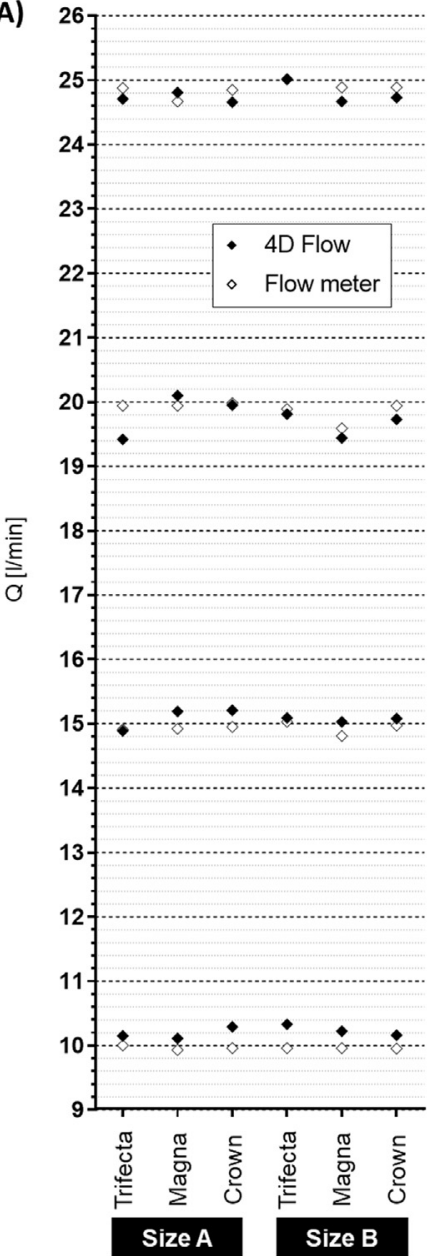

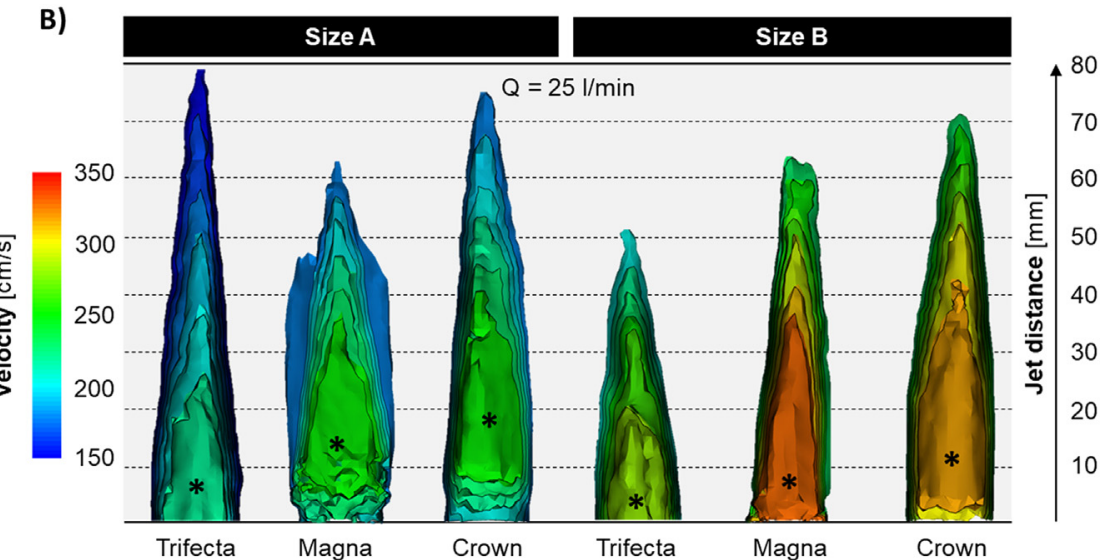

C)

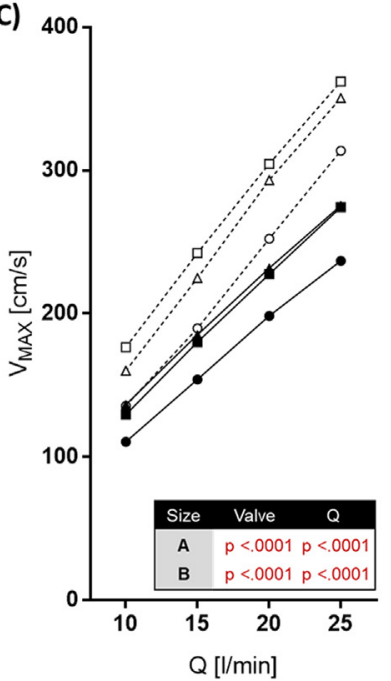

D)
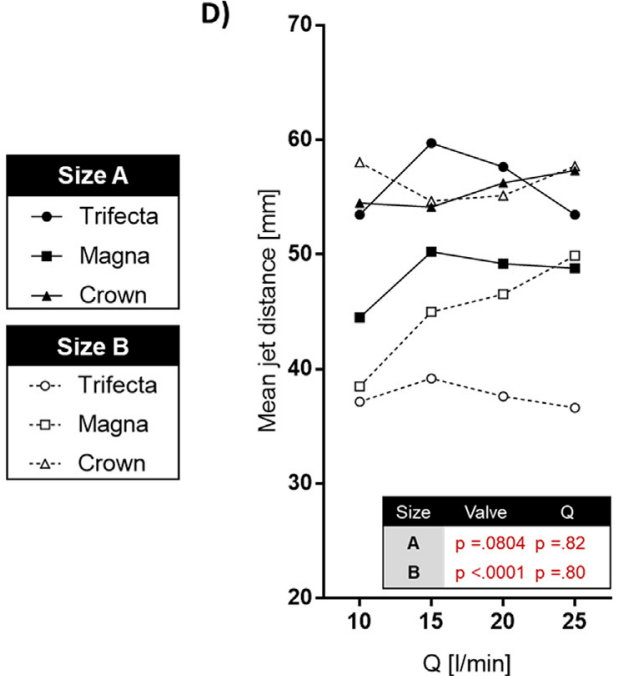

Fig. 4. BPV-specific 3D velocity flow pattern.

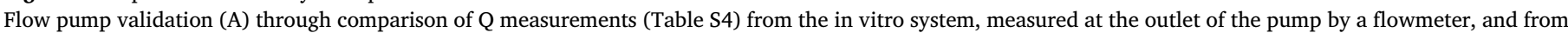

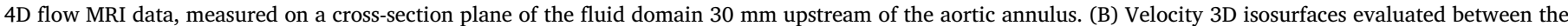

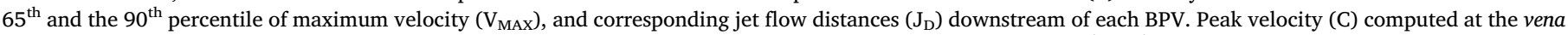
contracta, highlighted with a black asterisk (*), for each $4 \mathrm{D}$ Flow velocity field and mean $\mathrm{J}_{\mathrm{D}}$ averaged on the $65^{\text {th }}-90^{\text {th }} 3 \mathrm{D}$ velocity isosurfaces (D).

between 0.58 (size A tested at $10 \mathrm{l} / \mathrm{min}$, Fig. 7A) and 0.76 (size A tested at $25 \mathrm{l} / \mathrm{min}$, Fig. 7C). Notably, Trifecta size B outperformed both Crown and Magna, with the latter reporting the lowest overall $\mathrm{Pi}$ values.

\subsection{Transvalvular pressure drop}

For each BPV size, consistently with EOA estimations, Trifecta yielded the lowest TPG $\mathrm{MAX}_{\text {( }}$ (size $\mathrm{A}, p=0.0014$; size $\mathrm{B}, p=0.002$ ) and $\mathrm{TPG}_{\text {net }}$ (size A, $p=0.0145$; size $\mathrm{B}, p=0.0019$ ), respectively (Fig. 8A). Similar trends in pressure drops were observed in Magna and Crown $(p>0.24)$ at all the tested $\mathrm{Q}$, reporting $\mathrm{TPG}_{\mathrm{MAX}}$ and $\mathrm{TPG}_{\text {net }}$ up to 10 and $12 \mathrm{~mm} \mathrm{Hg}$ higher than Trifecta, respectively. Trifecta exhibited the lowest pressure recovery ( $p$ equal to 0.0168 and 0.0036 for size A and $\mathrm{B}$, respectively) if compared to Magna and Crown, which showed a similar behavior $(p>0.47)$. Both $\mathrm{TPG}_{\mathrm{MAX}}$ and $\mathrm{TPG}_{\text {net }}$ reported good to excellent correlation against ground-truth data from pressure transducers (Fig. 8B), i.e., $\mathrm{r}^{2}=0.96$ and $\mathrm{r}^{2}=0.89$, respectively. Bland-Altman bias (Fig. 9) was equal to $+0.09 \mathrm{~mm} \mathrm{Hg}$ for $\mathrm{TPG}_{\text {net }}$ (limits of agreements between $-4.60 \mathrm{~mm} \mathrm{Hg}$ and $+4.78 \mathrm{~mm} \mathrm{Hg}$ ) and $+1.13 \mathrm{~mm} \mathrm{Hg}$ for $\mathrm{TPG}_{\mathrm{MAX}}$ (limits of agreements from $-3.73 \mathrm{~mm} \mathrm{Hg}$ to $+5.99 \mathrm{~mm} \mathrm{Hg}$ ).

\subsection{Energy viscous dissipation}

BPVs were characterized by a similar KE $(p=0.2382)$ for size A (Fig. 10A), while differences appeared in size B $(p=0.0074)$ with Trifecta minimizing KE vs. Crown $(p=0.0867)$ and Magna ( $p=0.0077$ ). Considering the downstream $\dot{E}_{L}$ (Fig. 10B), BPVs showed a similar trend for size A, with slightly differences between Trifecta and Magna ( $p=0.0366)$. By contrast, for size $\mathrm{B}, \dot{E}_{L}$ was almost unaltered for Crown, which exhibited a significant lower degree of viscous dissipation up to $-66.3 \%$ and $-63.7 \%$ with respect to Magna $(p=0.0283)$ and Trifecta $(p=0.0105)$, respectively. Of note, power loss was inversely proportional to the fluid jet penetration (Fig. 3B): the lowest rate of viscous dissipation was paralleled by the highest $J_{\mathrm{D}}$ values as highlighted in the Trifecta size A $\left(\dot{E}_{L} \leq 0.57 \mathrm{~mW}\right.$ and $\mathrm{J}_{\mathrm{D}}$ up to $78 \mathrm{~mm})$ and in the Crown valve ( $\dot{E}_{L} \leq 0.50 \mathrm{~mW}$ and $\mathrm{J}_{\mathrm{D}}$ up to $75 \mathrm{~mm}$ in the size B). Conversely, the highest power loss was associated with the lowest jet penetration as reported at size B by both Trifecta $\left(\dot{E}_{L}\right.$ up to $1.50 \mathrm{~mW}$ and a maximum $\mathrm{J}_{\mathrm{D}}$ of $50.9 \mathrm{~mm}$ ) and Magna ( $\dot{E}_{L}$ up to $1.16 \mathrm{~mW}$ with a maximum $\mathrm{J}_{\mathrm{D}}$ of $62.2 \mathrm{~mm}$ ). Interestingly, Trifecta reported a very low viscous energy loss with the highest $J_{\mathrm{D}}$ for size $A$ and, conversely, a very high energy loss for size B paralleled by the lowest $\mathrm{J}_{\mathrm{D}}$. On the contrary, Crown reported the lowest $\dot{E}_{L}$ variations between size A and B. 
A)

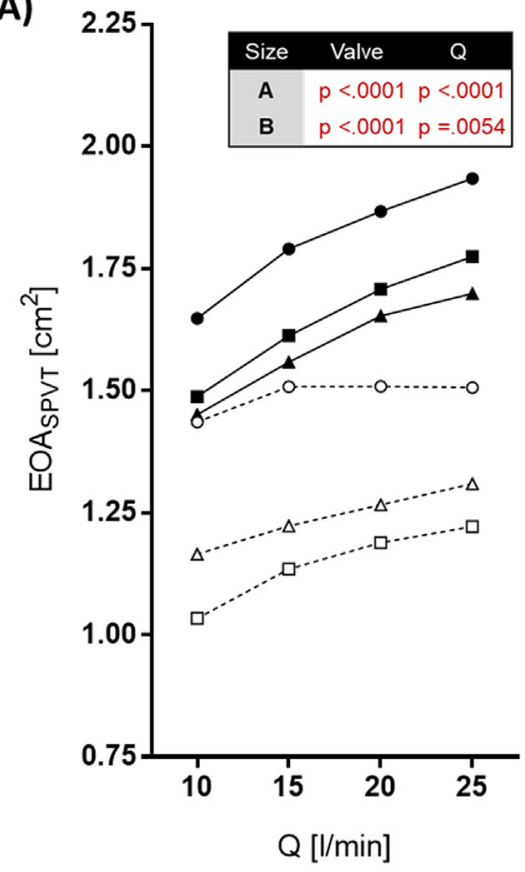

B)

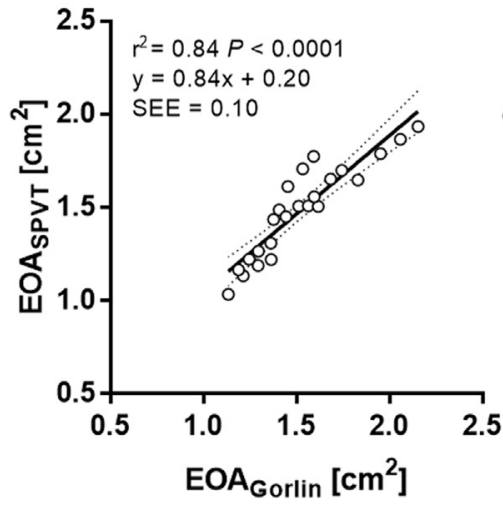

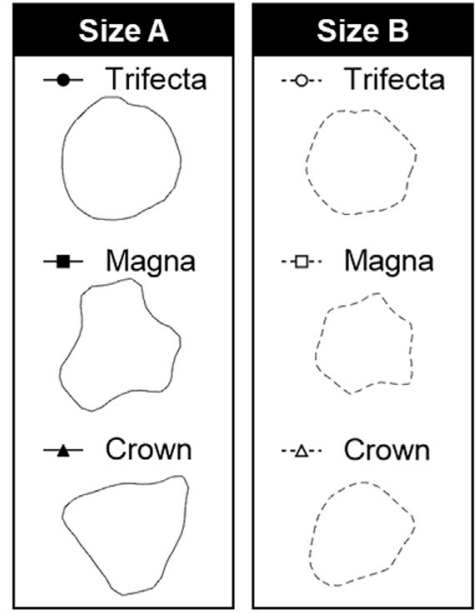

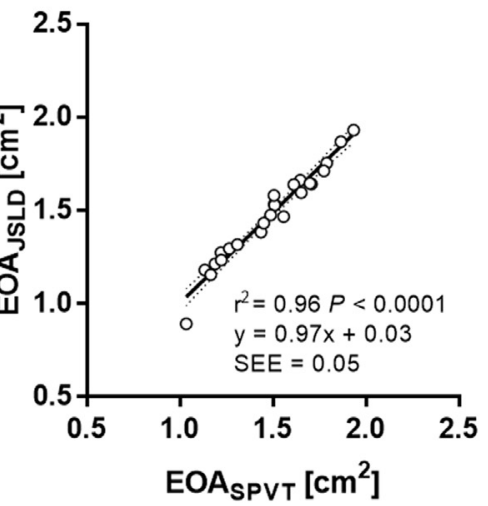

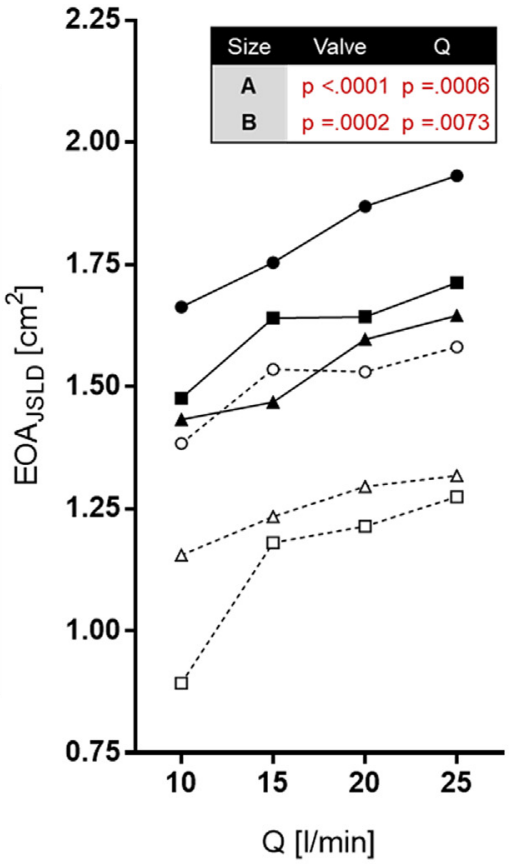

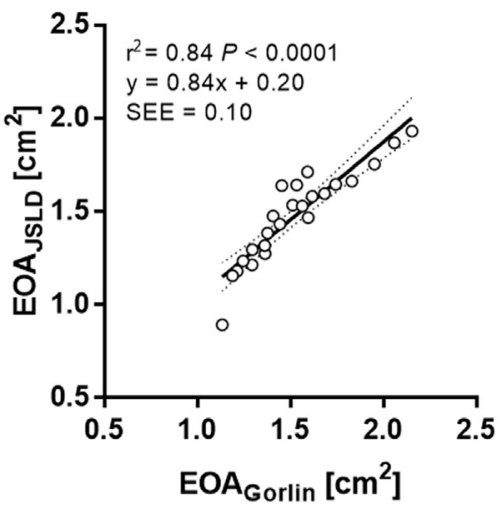

Fig. 5. Effective orifice area (EOA).

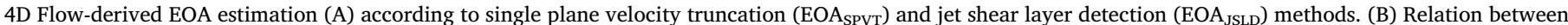

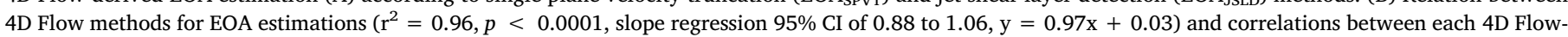

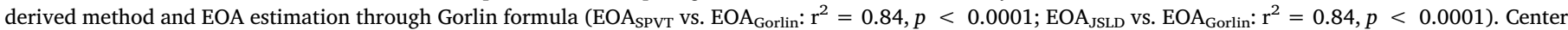

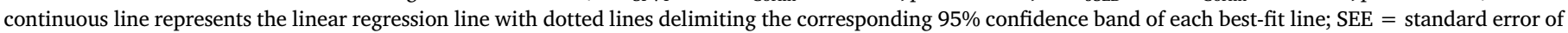
estimate; $\mathrm{CI}=$ confidence interval.

\section{Discussion}

In the present study, we demonstrated both the efficacy and the advantages of the proposed 4D Flow framework to comprehensively characterize the hemodynamics of three pericardial stented BPVs. Indeed, a robust comparative assessment of BPVs hemodynamic performances is crucial to tackle heterogeneous anatomical and hemodynamic requirements in guiding a patient-tailored BPV selection.

The 4D Flow acquisition effectively captures the 3D distribution of the velocity field and estimated blood pressure drops across each BPV while the MRI compatible in vitro setting assured a ground-truth term of comparison for TPG measurements.

According to our 4D Flow data, the hemodynamic performance of BPVs directly depends on their specific design. For instance, the externally mounted configuration of Trifecta leaflets assures an almost circular jet cross-section with minimal transvalvular pressure drops (i.e., $\mathrm{TPG}_{\mathrm{MAX}}$ and $\mathrm{TPG}_{\mathrm{net}}$ ) and the highest EOA within each BPV-size. Conversely, the internally mounted design of Magna leaflets significantly reduces the EOA available to blood flow, especially when considering size $\mathrm{B}$, mimicking a more pronounced PPM tendency.
Notably, Crown valve underperforms the Trifecta performance index, though designed with the bovine pericardium mounted outside the BPV stent.

Thanks to the 4D Flow full volumetric coverage of the velocity field, the comprehensive visualization of the $3 \mathrm{D}$ jet flow velocity structure is clearly detectable by using velocity isosurfaces (Fig. 4B). This facilitates the identification of the vena contracta and EOA calculation; 4D Flowdriven EOA measures proved to be consistent $\left(\mathrm{r}^{2}=0.96\right)$, reproducible, and in good agreement with in vitro EOA measures $\left(\mathrm{r}^{2}=0.84\right)$. Trifecta exhibited the highest EOA values, which were consistent with average EOAs reported at discharge in a large population of 1014 patients, i.e., $1.77 \mathrm{~cm}^{2}$ and $1.58 \mathrm{~cm}^{2}$ for BPV size A and B, respectively [32]. Also, 4D Flow EOA results are in agreement with pre-discharge echocardiographic results for 1436 implanted patients with Trifecta reporting higher EOA values, if compared to the other bovine bioprostheses, up to $1.84 \mathrm{~cm}^{2}$ and $1.53 \mathrm{~cm}^{2}$ for size A and B, respectively [33]. Of note, for all BPVs, our EOA results at different $Q$ flow rates confirm an EOA flow dependency, as already observed on pericardial stented valves by previous experimental analyses [5,34].

The elaboration of 4D Flow data yielded reliable estimations of 3D 
A)

$E_{\text {JSLD }}$ vs EOA
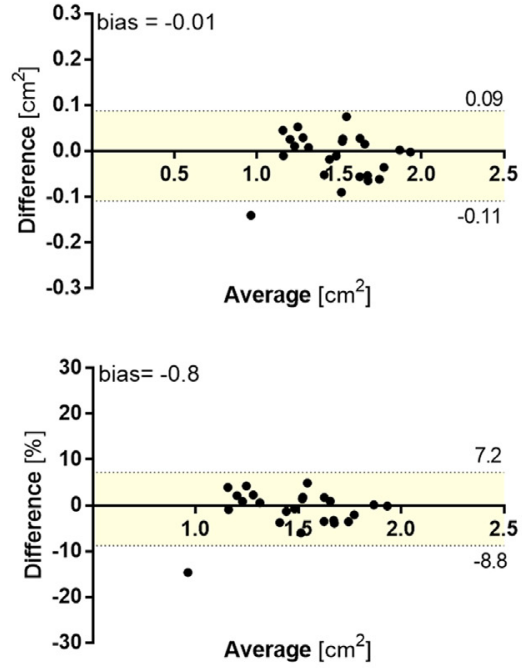

B)

$\mathrm{EOA}_{\mathrm{SPVT}}$ vs $\mathrm{EOA}_{\text {Gorlin }}$
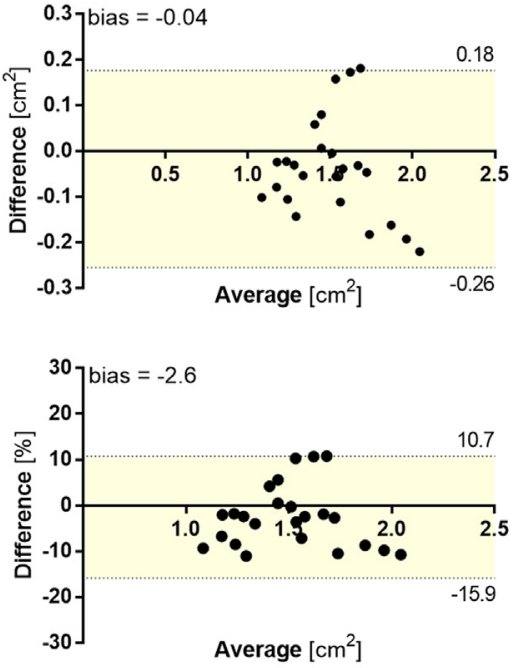

C)

EOA $_{\text {JSLD }}$ vs EOA Gorlin

Fig. 6. Bland-Altman plots of EOA differences.

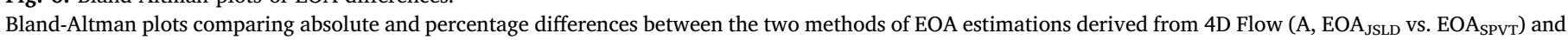

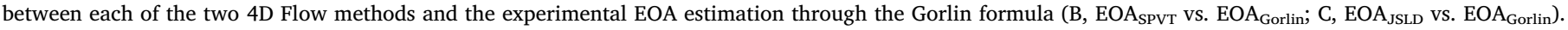
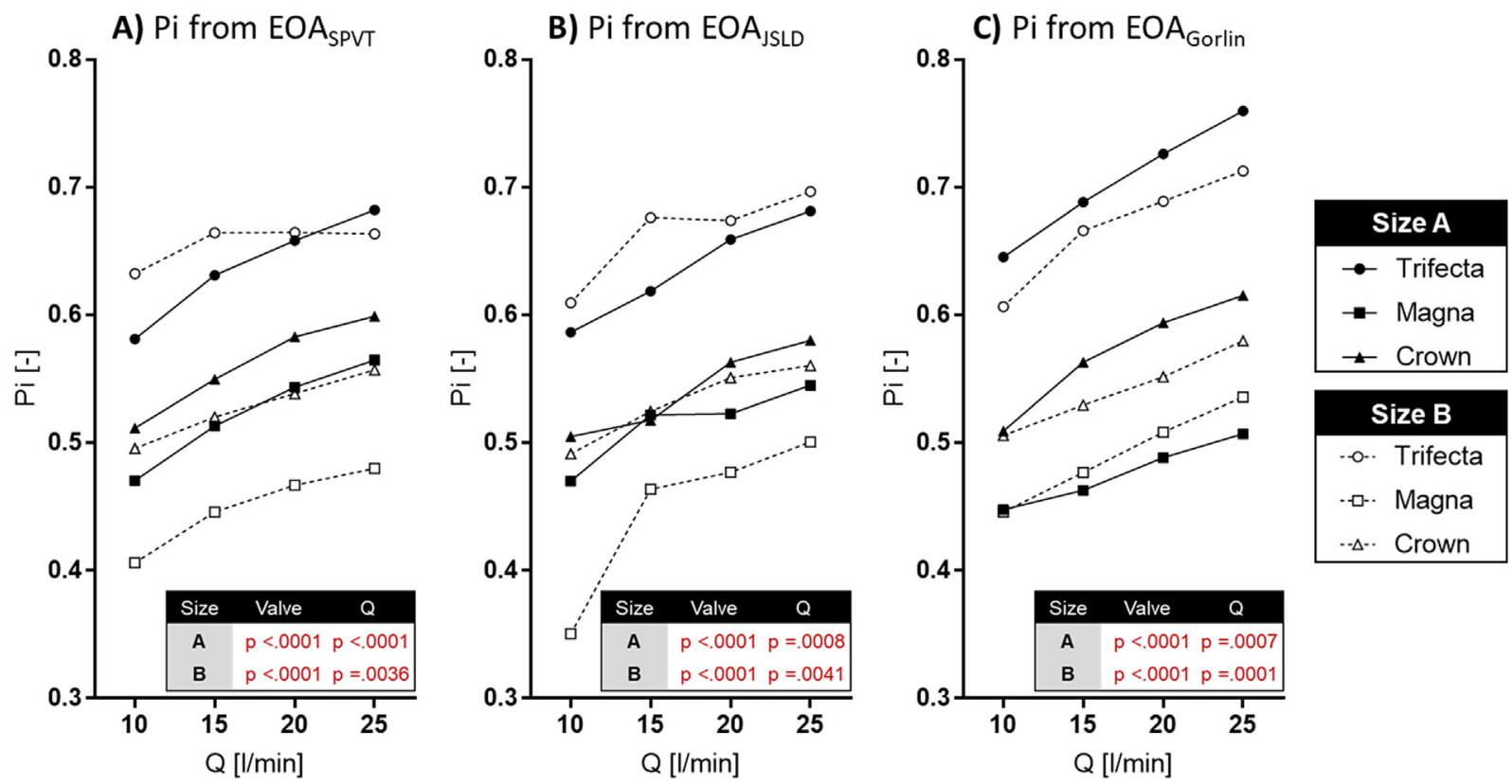

Fig. 7. BPVs performance index.

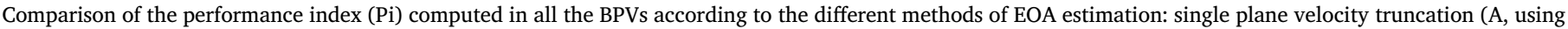

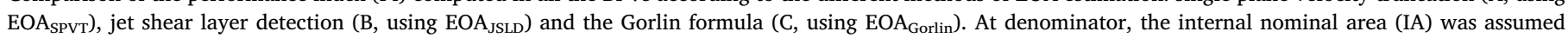
circular and calculated according to the nominal internal diameter (ID) of each tested BPV (Table 1).

relative pressure field within the acquired volume. 4D Flow $T_{P G} G_{M A X}$ and $\mathrm{TPG}_{\text {net }}$ matched well with the corresponding measurements taken from pressure transducers $\left(r^{2}=0.96\right.$ for TPG ${ }_{\mathrm{MAX}}$ and $\mathrm{r}^{2}=0.89$ for $\mathrm{TPG}_{\text {net }}$ ), effectively capturing the pressure recovery phenomenon downstream of each BPV. In accordance with clinical studies [33,35], our data corroborate the hemodynamic differences observed between pericardial BPVs and confirm the lowest mean gradients $(p<0.01)$ reported for Trifecta in early postoperative conditions [33]. At 6-month follow-up [35], the Trifecta valve still exhibited higher aortic valve area than Magna but no significant influence of BPV type on pressure gradients could be demonstrated. Within the clinical community, a longer follow-up is still expected, especially for the Trifecta valve [36,37], in order to elucidate whether these small but consistent differences can have a tangible impact on mid- to long-term clinical outcomes.

The proposed 4D Flow hemodynamic comparison can directly impact aspects of potential clinical relevance.

First, the 4D Flow analysis is valid per se to enable effective and independent in vitro evaluations of BPVs hemodynamic performance under repeatable and fully controlled conditions $[5,13,35]$.

Second, the potential of 4D Flow analysis is directly transferable to clinical in vivo scenarios enabling the 3D comprehensive assessment of patient-specific aortic hemodynamics, including EOA calculation and 

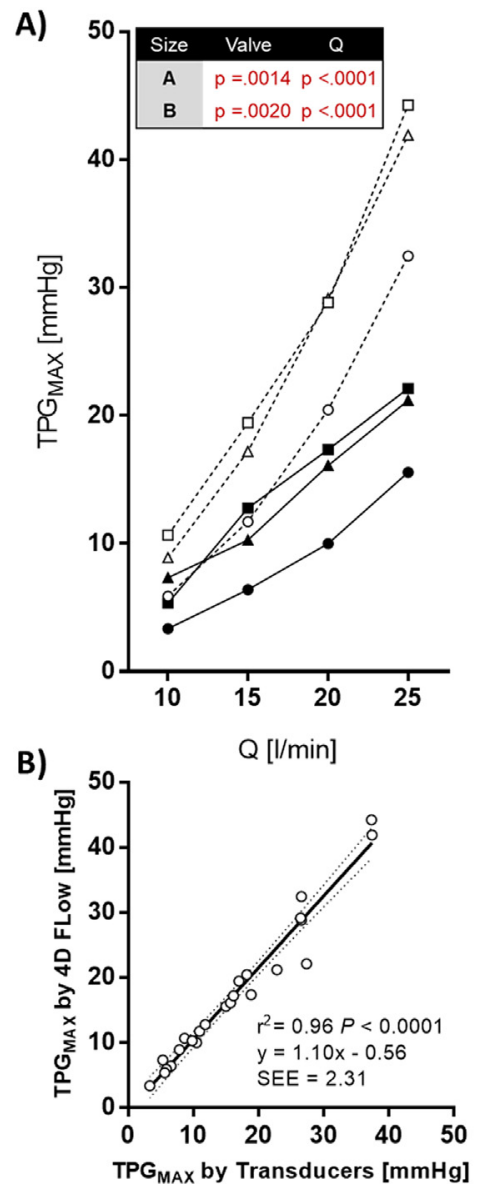
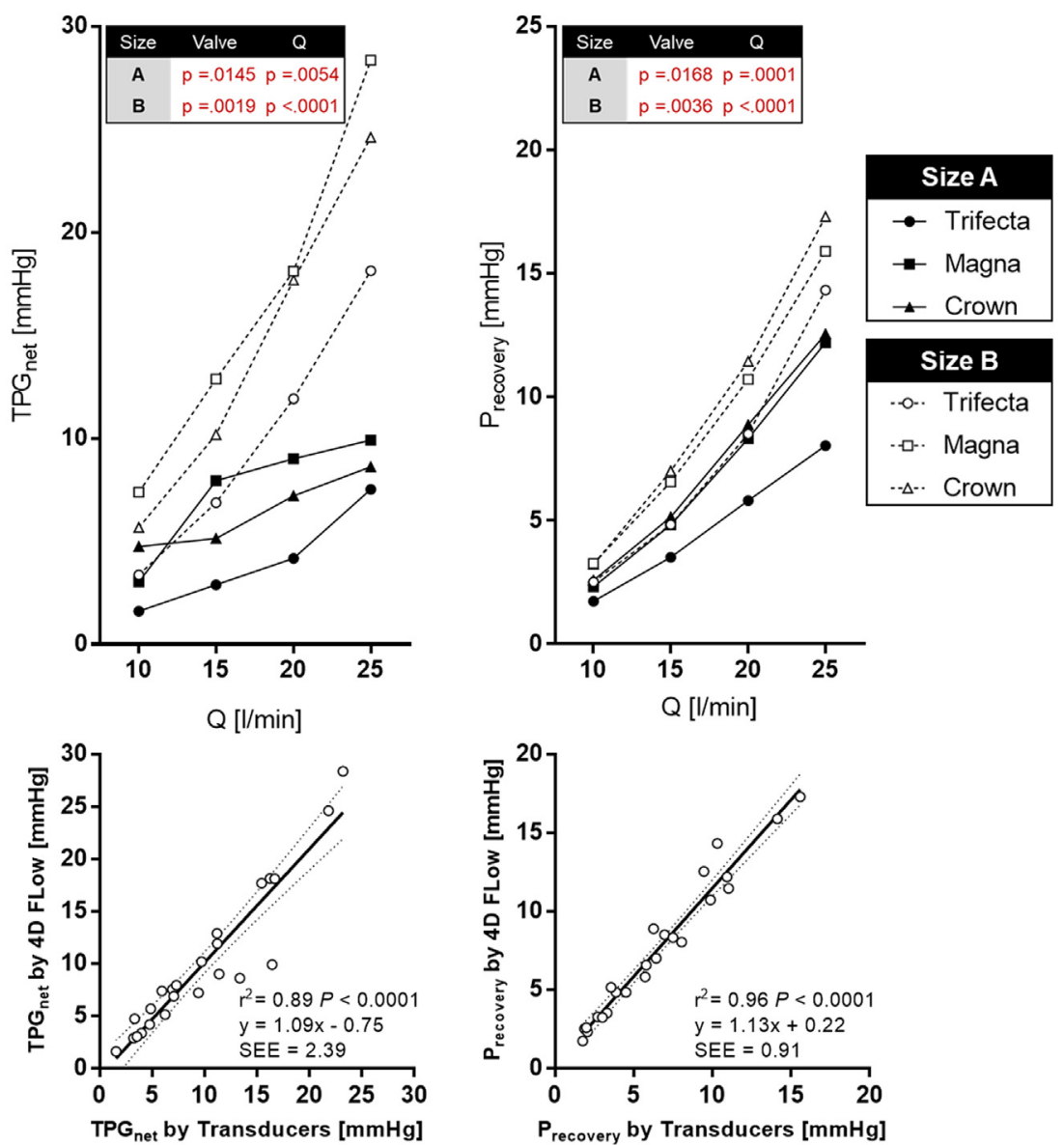

Fig. 8. Transvalvular pressure drop.

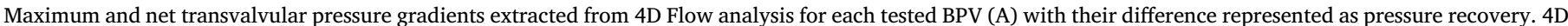

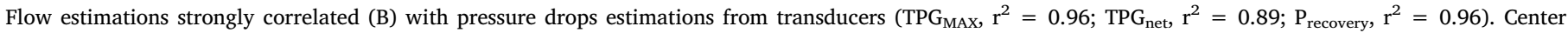

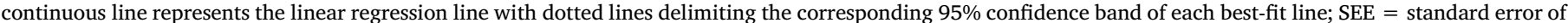
estimate; $\mathrm{CI}=$ confidence interval.

A)

TPG ${ }_{\text {MAX }}$

4D Flow vs Transducers
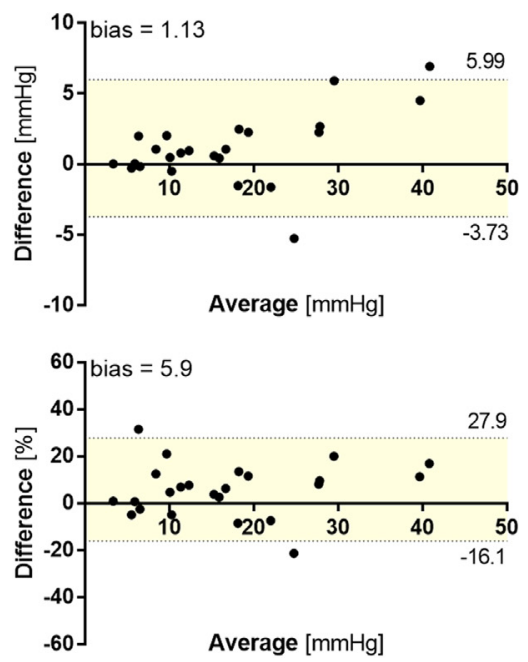

B)

4D Flow vs Transducers
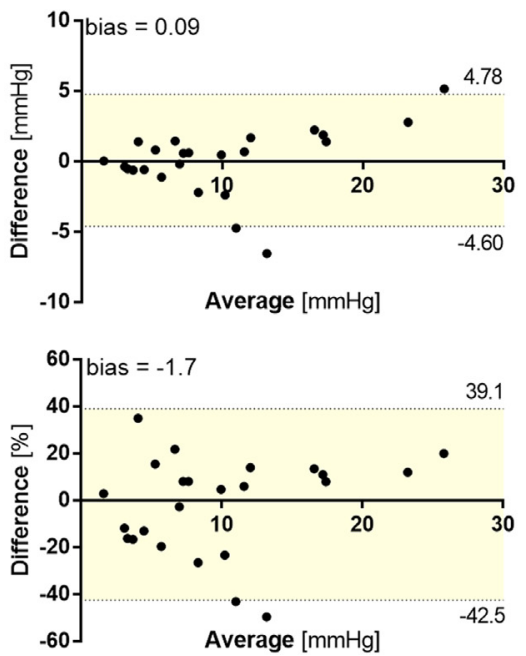

C) Pressure recovery 4D Flow vs Transducers
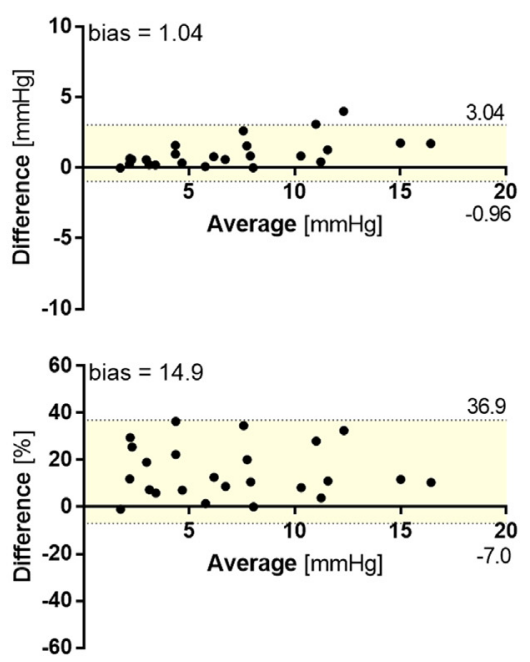

Fig. 9. Bland-Altman plots of TPG differences.

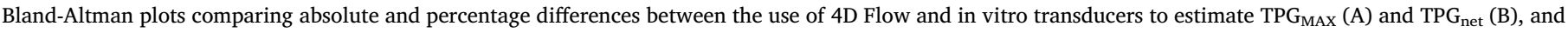
their difference, i.e., pressure recovery (C). 

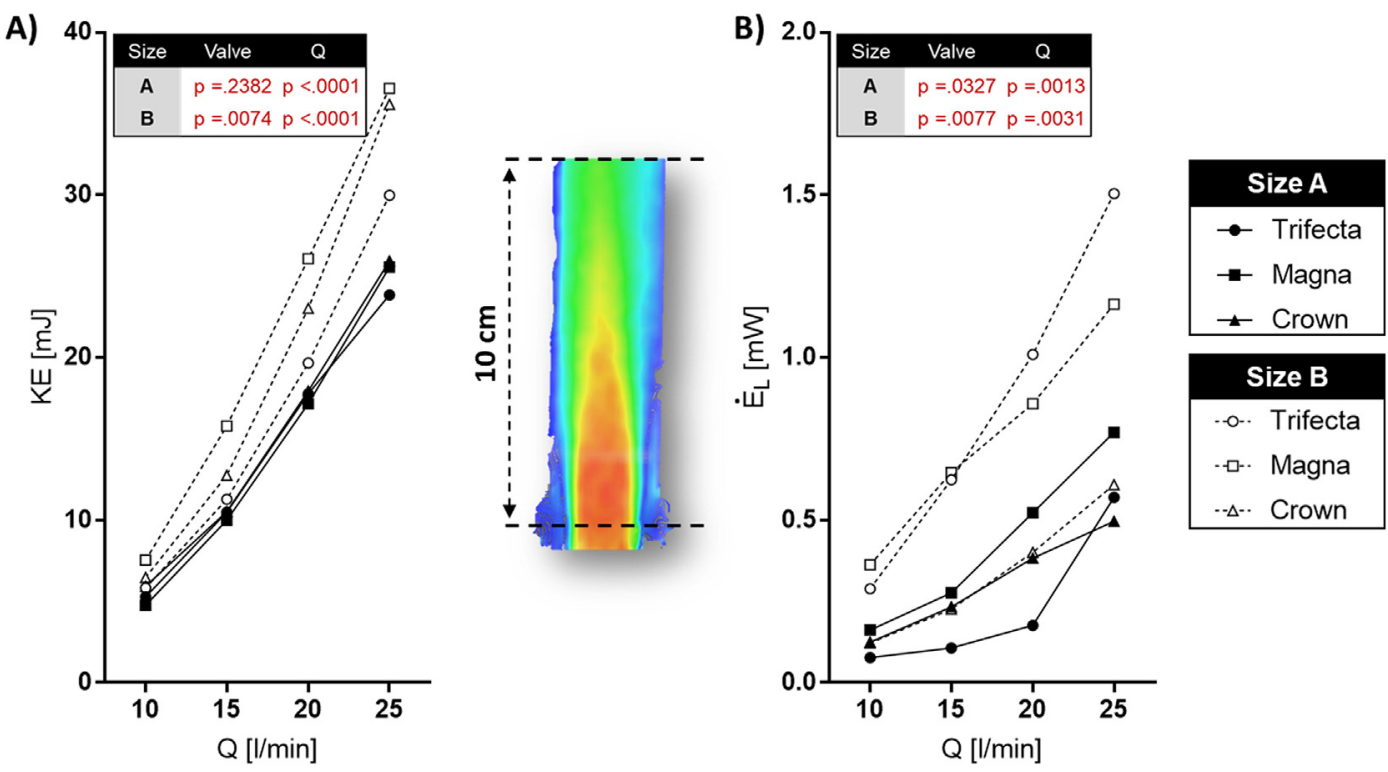

Fig. 10. BPV-specific energetics.

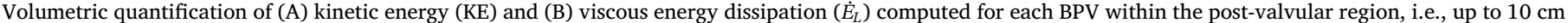
downstream of each BPV along the longitudinal axis of the 3D-printed AR model.

non-invasive TPG spatial mapping. On the one hand, robust EOA quantification is crucial for PPM assessment, clinically identified by means of the indexed EOA (EOAI), defined as the ratio between the EOA of a normally functioning prosthesis and the patient body surface area [6]. For instance, the largest EOA provided by Trifecta valve is associated with a low incidence of severe PPM [32] and an overall higher EOAI $(p<0.001)$ at discharge [33]. On the other hand, the advent of non-invasive 4D Flow mapping of TPGs within the AR can be extremely beneficial in the preoperative diagnosis of aortic stenosis severity providing information complementary to conventional echocardiography [38], although the assumption of laminar Newtonian fluid flow still limits its use in highly stenotic turbulent flows [12]. Both small BPVs size and PPM have been associated with increased TPGs across BPVs [6,39].

Third, 4D Flow offers an additional insight into blood flow energetics as represented by viscous energy dissipation $\left(\dot{E}_{L}\right)$, which proved able to capture the different 3D flow peculiarities of the three tested BPVs and the not negligible effect of BPV size (Fig. 10B). Of note, $\dot{E}_{L}$ measurements suggest that the Trifecta hemodynamics is more sensitive than Magna and Crown to a variation in the device size; both size and design of each BPV may be relevant factors able to trigger the generation of frictional forces and differentiate the fluid evolution in the AR site of implantation.

In particular, $\dot{E}_{L}$ can provide an estimation of aortic flow inefficiencies associated with aortic stenosis [30], and it may prove useful for the functional assessment of BPVs during the follow-up. Indeed, fibrosis and calcification are the predominant modes of structural valve degeneration (SVD) in pericardial BPVs leading to aortic stenosis [40]; in this scenario, $\dot{E}_{L}$ could represents a more sensitive marker, than conventional parameters, of subtle alterations eventually preceding more evident features of valve degeneration.

\section{Limitations}

Four limitations of the present analysis should be taken into consideration when interpreting results.

First, steady flow conditions were employed to assess BPV hemodynamics, thus neglecting the influence of flow pulsatility. Though reducing the complexity of the study, the use of steady flow tests proved able to magnify the energy losses of prosthetic valves at the peak systolic velocity of a physiological pulsatile flow regime [41,42] and allowed to examine the effect of valve shape and flow rate [43]. To enable pulsatile flow conditions, the in vitro system will be equipped with a pulsatile MR-compatible pump unit $[14,44]$ and an adjustable hydraulic afterload mimicking the hydraulic input impedance of the system circulation [45].

Second, we employed a rigid AR phantom which did not allow to reproduce AR compliance, which is irrelevant in steady flow conditions. For future pulsatile flow testing purpose, flexible 3D-printed or silicone AR models could be used [44,46]. Also, a symmetrical AR anatomy was assumed and coronary perfusion was not modelled in the in vitro system [47].

Third, monitoring in vitro the BPV deterioration progression is unlikely as the single BPV could be tested no more than $1 \mathrm{~h}$. In the future, by applying in vitro calcification protocols [48], the BVPs could be tested at different timepoints of the progressing calcification process. Also, extending the present 4D Flow protocol to in vivo analysis could definitely enable to monitor BPV at the different stages of structural valve deterioration [49]. Indeed, extensive 4D Flow longitudinal data may further elucidate the biomechanical impact of BPV-related factors on SVD incidence, also addressing the urgent need for standard definitions to provide accurate data on SVD [3].

Fourth, 4D Flow results were validated against ground-truth experimental data without considering postoperative in vivo 4D Flow data from patients receiving the tested BPVs. Though beyond the aim of the present study, 4D Flow analysis is ongoing on real patients referred to BPV implantation at our hospital centre.

\section{Conclusion}

The present MRI-compatible benchmark protocol demonstrated the 4D Flow capability to standardize the comparison of BPVs hydrodynamic performance; further extension to BPVs types and sizes could provide overall benchmark values to comprehensive compare commercialized BPVs. The protocol could also support pre-clinical assessment of prototypal cardiac valves [50] and potentially reduce the need for animal testing [51]. 


\section{Sources of funding statement}

The study investigators and their institutions received no funding for conducting this study.

\section{CRediT authorship contribution statement}

Francesco Sturla:Conceptualization, Methodology, Investigation, Formal analysis, Writing - original draft, Writing review \& editing, Project administration.Filippo Piatti:Conceptualization, Methodology, Software, Investigation, Writing - original draft, Writing - review \& editing.Michal Jaworek:Methodology, Investigation, Writing - review \& editing.Federico Lucherini:Methodology, Investigation, Data curation, Writing - review \& editing.Francesca R. Pluchinotta:Methodology, Investigation, Data curation, Writing review \& editing.Sergii V. Siryk:Software, Validation, Writing - review \& editing.Daniel Giese:Data curation, Writing - review \& editing.Riccardo Vismara:Validation, Writing - review \& editing.Giordano Tasca:Data curation, Visualization, Writing - review \& editing.Lorenzo Menicanti:Visualization, Funding acquisition, Writing - review \& editing.Alberto Redaelli:Funding acquisition, Resources, Writing - review \& editing.Massimo Lombardi:Funding acquisition, Resources, Supervision, Writing review \& editing.

\section{Declaration of competing interest}

None of the authors have conflicts of interest to declare.

\section{Acknowledgements}

This work has been supported by IRCCS Policlinico San Donato, a clinical research hospital partially funded by the Italian Ministry of Health. The authors thank Denis Tresova and Elvis Tresova for their valuable assistance in 4D Flow data acquisitions.

\section{Appendix A. Supplementary data}

Supplementary data to this article can be found online at https:// doi.org/10.1016/j.mri.2020.01.006.

\section{References}

[1] Brown JM, O'Brien SM, Wu C, Sikora JAH, Griffith BP, Gammie JS. Isolated aortic valve replacement in North America comprising 108,687 patients in 10 years: changes in risks, valve types, and outcomes in the Society of Thoracic Surgeons National Database. J Thorac Cardiovasc Surg 2009;137(1):82-90.

[2] Andell P, Li X, Martinsson A, Andersson C, Stagmo M, Zöller B, et al. Epidemiology of valvular heart disease in a Swedish nationwide hospital-based register study. Heart 2017;103(21):1696-703.

[3] Rodriguez-Gabella T, Voisine P, Puri R, Pibarot P, Rodés-Cabau J. Aortic bioprosthetic valve durability. Incidence, Mechanisms, Predictors, and Management of Surgical and Transcatheter Valve Degeneration 2017;70(8):1013-28.

[4] Doenst T, Amorim PA, Al-Alam N, Lehmann S, Mukherjee C, Faerber G. Where is the common sense in aortic valve replacement? A review of hemodynamics and sizing of stented tissue valves. J Thorac Cardiovasc Surg 2011;142(5):1180-7.

[5] Gerosa G, Tarzia V, Rizzoli G, Bottio T. Small aortic annulus: the hydrodynamic performances of 5 commercially available tissue valves. J Thorac Cardiovasc Surg 2006;131(5):1058-64.

[6] Pibarot P, Dumesnil JG. Prosthetic heart valves: selection of the optimal prosthesis and long-term management. Circulation 2009;119(7):1034-48.

[7] Lancellotti P, Pibarot P, Chambers J, Edvardsen T, Delgado V, Dulgheru R, et al. Recommendations for the imaging assessment of prosthetic heart valves: a report from the European Association of Cardiovascular Imaging endorsed by the Chinese Society of Echocardiography, the Inter-American Society of Echocardiography, and the Brazilian Department of Cardiovascular Imaging †. Eur Heart J Cardiovasc Imaging 2016;17(6):589-90.

[8] Botzenhardt F, Eichinger WB, Bleiziffer S, Guenzinger R, Wagner IM, Bauernschmitt $\mathrm{R}$, et al. Hemodynamic comparison of bioprostheses for complete supra-annular position in patients with small aortic annulus. J Am Coll Cardiol 2005;45(12):2054-60.
[9] Pibarot P, Dumesnil JG. Prosthesis-patient mismatch: definition, clinical impact, and prevention. Heart 2006;92(8):1022-9.

[10] Suri RM, Michelena HI, Burkhart HM, Greason KL, Daly RC, Dearani JA, et al. A prospective, randomized comparison of 3 contemporary bioprosthetic aortic valves: should hemodynamic performance influence device selection? J Thorac Cardiovasc Surg 2012;144(6):1387-95. 98. discussion 95-7.

[11] Dyverfeldt P, Bissell M, Barker AJ, Bolger AF, Carlhäll C-J, Ebbers T, et al. 4D flow cardiovascular magnetic resonance consensus statement. J Cardiovasc Magn Reson 2015;17(1):72.

[12] Garcia J, Barker AJ, Markl M. The role of imaging of flow patterns by 4D Flow MRI in aortic stenosis. JACC Cardiovasc Imaging 2019;12(2):252-66.

[13] Tasca G, Vismara R, Fiore GB, Mangini A, Romagnoni C, Pelenghi S, et al. Fluiddynamic results of in vitro comparison of four pericardial bioprostheses implanted in small porcine aortic roots. Eur J Cardiothorac Surg 2015;47(2):e62-7.

[14] von Knobelsdorff-Brenkenhoff F, Dieringer MA, Greiser A, Schulz-Menger J. In vitro assessment of heart valve bioprostheses by cardiovascular magnetic resonance: four-dimensional mapping of flow patterns and orifice area planimetry. Eur J Cardiothorac Surg 2011;40(3):736-42.

[15] Kvitting JP, Dyverfeldt P, Sigfridsson A, Franzen S, Wigstrom L, Bolger AF. In vitro assessment of flow patterns and turbulence intensity in prosthetic heart valves using generalized phase-contrast MRI. J Magn Reson Imaging 2010;31.

[16] Garcia J, Markl M, Schnell S, Allen B, Entezari P, Mahadevia R, et al. Evaluation of aortic stenosis severity using 4D flow jet shear layer detection for the measurement of valve effective orifice area. Magn Reson Imaging 2014;32(7):891-8.

[17] Krittian SB, Lamata P, Michler C, Nordsletten DA, Bock J, Bradley CP, et al. A finiteelement approach to the direct computation of relative cardiovascular pressure from time-resolved MR velocity data. Med Image Anal 2012;16(5):1029-37.

[18] Bock J, Frydrychowicz A, Lorenz R, Hirtler D, Barker AJ, Johnson KM. In vivo noninvasive 4D pressure difference mapping in the human aorta: phantom comparison and application in healthy volunteers and patients. Magn Reson Med 2011;66.

[19] Bach DS. Echo/Doppler evaluation of hemodynamics after aortic valve replacement: principles of interrogation and evaluation of high gradients. JACC Cardiovasc Imaging 2010;3.

[20] Piatti F, Palumbo MC, Consolo F, Pluchinotta F, Greiser A, Sturla F, et al. Experimental quantification of the fluid dynamics in blood-processing devices through 4D-flow imaging: a pilot study on a real oxygenator/heat-exchanger module. J Biomech 2018;68:14-23.

[21] Piatti F, Sturla F, Bissell MM, Pirola S, Lombardi M, Nesteruk I, et al. 4D Flow analysis of BAV-related fluid-dynamic alterations: evidences of wall shear stress alterations in absence of clinically-relevant aortic anatomical remodeling. Front Physiol 2017:8(441).

[22] Garcia J, Marrufo OR, Rodriguez AO, Larose E, Pibarot P, Kadem L. Cardiovascular magnetic resonance evaluation of aortic stenosis severity using single plane measurement of effective orifice area. J Cardiovasc Magn Reson 2012;14:23.

[23] Kadem L, Knapp Y, Pibarot P, Bertrand E, Garcia D, Durand LG, et al. A new experimental method for the determination of the effective orifice area based on the acoustical source term. Exp Fluids 2005;39(6):1051.

[24] Borisyuk AO. Experimental study of noise produced by steady flow through a simulated vascular stenosis. J Sound Vib 2002;256(3):475-98.

[25] Garcia D, Dumesnil JG, Durand LG, Kadem L, Pibarot P. Discrepancies between catheter and Doppler estimates of valve effective orifice area can be predicted from the pressure recovery phenomenon: practical implications with regard to quantification of aortic stenosis severity. J Am Coll Cardiol 2003;41(3):435-42.

[26] Toger J, Arvidsson PM, Bock J, Kanski M, Pedrizzetti G, Carlsson M, et al. Hemodynamic forces in the left and right ventricles of the human heart using 4D flow magnetic resonance imaging: phantom validation, reproducibility, sensitivity to respiratory gating and free analysis software. Plos One 2018;13(4):e0195597.

[27] Saitta S, Pirola S, Piatti F, Votta E, Lucherini F, Pluchinotta F, et al. Evaluation of 4D flow MRI-based non-invasive pressure assessment in aortic coarctations. J Biomech. 2019.

[28] Richau J, Dieringer MA, Traber J, von Knobelsdorff-Brenkenhoff F, Greiser A Schwenke C, et al. Effects of heart valve prostheses on phase contrast flow measurements in cardiovascular magnetic resonance - a phantom study. J Cardiovasc Magn Reson 2017;19(1):5.

[29] Crandon S, Westenberg JJM, Swoboda PP, Fent GJ, Foley JRJ, Chew PG, et al. Impact of age and diastolic function on novel, 4D flow CMR biomarkers of left ventricular blood flow kinetic energy. Sci Rep 2018;8(1):14436.

[30] Barker AJ, van Ooij P, Bandi K, Garcia J, Albaghdadi M, McCarthy P, et al. Viscous energy loss in the presence of abnormal aortic flow. Magn Reson Med 2014;72(3):620-8.

[31] Venkatachari AK, Halliburton SS, Setser RM, White RD, Chatzimavroudis GP. Noninvasive quantification of fluid mechanical energy losses in the total cavopulmonary connection with magnetic resonance phase velocity mapping. Magn Reson Imaging 2007;25(1):101-9.

[32] Bavaria JE, Desai ND, Cheung A, Petracek MR, Groh MA, Borger MA, et al. The St Jude Medical Trifecta aortic pericardial valve: results from a global, multicenter, prospective clinical study. J Thorac Cardiovasc Surg 2014;147(2):590-7.

[33] Ugur M, Suri RM, Daly RC, Dearani JA, Park SJ, Joyce LD, et al. Comparison of early hemodynamic performance of 3 aortic valve bioprostheses. J Thorac Cardiovasc Surg 2014;148(5):1940-6.

[34] Hartrumpf M, Kuehnel R-U, Puchner R, Albes JM, Pohl A, Pohl M, et al. Characteristic resistance curves of aortic valve substitutes facilitate individualized decision for a particular type. Eur J Cardiothorac Surg 2005;27(3):450-5.

[35] Wendt D, Thielmann M, Plicht B, Assmann J, Price V, Neuhauser M, et al. The new St Jude Trifecta versus Carpentier-Edwards Perimount Magna and Magna Ease 
aortic bioprosthesis: is there a hemodynamic superiority? J Thorac Cardiovasc Surg 2014;147(5):1553-60.

[36] Emery RW, Krogh CC. The show must go on. J Thorac Cardiovasc Surg 2017; 153(3):570.

[37] Goldman S, Cheung A, Bavaria JE, Petracek MR, Groh MA, Schaff HV. Midterm, multicenter clinical and hemodynamic results for the Trifecta aortic pericardial valve. J Thorac Cardiovasc Surg 2017;153(3). [561-9.e2].

[38] Baumgartner H, Hung J, Bermejo J, Chambers JB, Edvardsen T, Goldstein S, et al. Recommendations on the echocardiographic assessment of aortic valve stenosis: a focused update from the European Association of Cardiovascular Imaging and the American Society of Echocardiography. J Am Soc Echocardiogr 2017;30(4):372-92.

[39] Flameng W, Herregods MC, Vercalsteren M, Herijgers P, Bogaerts K, Meuris B. Prosthesis-patient mismatch predicts structural valve degeneration in bioprosthetic heart valves. Circulation 2010;121(19):2123-9.

[40] Grunkemeier GL, Furnary AP, Wu Y, Wang L, Starr A. Durability of pericardial versus porcine bioprosthetic heart valves. J Thorac Cardiovasc Surg 2012;144(6):1381-6.

[41] Salica A, Pisani G, Morbiducci U, Scaffa R, Massai D, Audenino A, et al. The combined role of sinuses of valsalva and flow pulsatility improves energy loss of the aortic valve. Eur J Cardiothorac Surg 2016;49(4):1222-7.

[42] Yellin EL, Peskin CS. Large amplitude pulsatile water flow across an orifice. J Dyn Syst Meas Control 1975;97(1):92-5.

[43] Ha H, Kvitting JP, Dyverfeldt P, Ebbers T. Validation of pressure drop assessment using 4D flow MRI-based turbulence production in various shapes of aortic stenoses. Magn Reson Med 2019;81(2):893-906.
[44] Urbina J, Sotelo JA, Springmuller D, Montalba C, Letelier K, Tejos C, et al. Realistic aortic phantom to study hemodynamics using MRI and cardiac catheterization in normal and aortic coarctation conditions. J Magn Reson Imaging 2016;44(3):683-97.

[45] Vismara R, Fiore GB, Mangini A, Contino M, Lemma M, Redaelli A, et al. A novel approach to the in vitro hydrodynamic study of the aortic valve: mock loop development and test. ASAIO J 2010;56(4):279-84.

[46] Butera G, Sturla F, Pluchinotta FR, Caimi A, Carminati M. Holographic augmented reality and 3D printing for advanced planning of sinus venosus ASD/partial anomalous pulmonary venous return percutaneous management. J Am Coll Cardiol Intv 2019;12(14):1389-91.

[47] Piola M, Vismara R, Tasca G, Lucherini F, Redaelli P, Soncini M, et al. Design of a simple coronary impedance simulator for the in vitro study of the complex coronary hemodynamics. Physiol Meas 2016;37(12):2274-85.

[48] Pettenazzo E, Deiwick M, Thiene G, Molin G, Glasmacher B, Martignago F, et al. Dynamic in vitro calcification of bioprosthetic porcine valves: evidence of apatite crystallization. J Thorac Cardiovasc Surg 2001;121(3):500-9.

[49] Salaun E, Clavel MA, Rodes-Cabau J, Pibarot P. Bioprosthetic aortic valve durability in the era of transcatheter aortic valve implantation. Heart 2018;104(16):1323-32.

[50] Zhang BL, Bianco RW, Schoen FJ. Preclinical assessment of cardiac valve substitutes: current status and considerations for engineered tissue heart valves. Front Cardiovasc Med 2019;6. 72-.

[51] Hubrecht RC, Carter E. The 3Rs and humane experimental technique: implementing change. Animals (Basel) 2019;9(10):754. 